\title{
XMM-Newton observation of the Tycho supernova remnant ${ }^{\star}$
}

\author{
A. Decourchelle ${ }^{1}$, J. L. Sauvageot ${ }^{1}$, M. Audard ${ }^{2}$, B. Aschenbach ${ }^{3}$, S. Sembay ${ }^{4}$, R. Rothenflug ${ }^{1}$, J. Ballet ${ }^{1}$, \\ T. Stadlbauer ${ }^{3}$, and R. G. West ${ }^{4}$ \\ 1 Service d'Astrophysique, CEA Saclay, 91191 Gif-sur-Yvette Cedex, France \\ 2 Paul Scherrer Institute, Laboratory for Astrophysics, 5232 Villigen PSI, Switzerland \\ 3 MPI für extraterrestrische Physik, 85741 Garching, Germany \\ ${ }^{4}$ Department of Physics and Astronomy, Leicester University, Leicester LE1 7RH, UK
}

Received 6 October 2000 / Accepted 6 November 2000

\begin{abstract}
We present the observation of the Tycho supernova remnant obtained with the EPIC and RGS instruments onboard the XMM-Newton satellite. We compare images and azimuthally averaged radial profiles in emission lines from different elements (silicon and iron) and different transition lines of iron (Fe L and Fe K). While the Fe XVII L line and Si XIII K line images are globally spatially coincident, the Fe K emission clearly peaks at a smaller radius, indicating a higher temperature toward the reverse shock. This is qualitatively the profile expected when the reverse shock, after travelling through the outer power-law density profile, has entered the central plateau of the ejecta. The high energy continuum map has an overall smooth distribution, with a similar extent to the radio emission. Its radial profile peaks further out than the lines emission. Brighter and harder continuum regions are observed with a rough bipolar symmetry in the eastern and western edges. The spectral analysis of the southeastern knots supports spatial variations of the relative abundance of silicon and iron, which implies an incomplete mixing of the silicon and iron layers.
\end{abstract}

Key words. ISM: supernova remnants - shock waves - supernovae: individual: Tycho - X-rays: ISM

\section{Introduction}

The Tycho supernova remnant (SNR) is considered as the prototype for type Ia remnants (Baade 1945). The supernova was recorded in 1572 by Tycho Brahe, hence, the remnant is relatively young and still retains much information about the progenitor. Its X-ray emission is dominated by shocked ejecta. With an angular diameter of 8 arcmin, Tycho SNR is well suited for investigating the spatial distribution of heavy elements in the ejecta. Hwang \& Gotthelf (1997) presented deconvolved line images of the object using $A S C A$ data. They found that the Fe $\mathrm{K}$ radial profile peaks at smaller radius than the other emission lines. This was interpreted as a possible iron stratification in the interior of the ejecta.

With XMM-Newton observatory, it is now possible to perform true spatially resolved spectroscopy of Tycho SNR, allowing the mapping of the emission for individual elements (and individual ions) and for the continuum

Send offprint requests to: A. Decourchelle;

e-mail: decour@calix.saclay.cea.fr

* Based on an observation obtained with XMM-Newton, an ESA science mission with instruments and contributions directly funded by ESA Member States and the USA (NASA). at high energy. In this paper we present a first study of Tycho SNR with XMM-Newton.

\section{Observation}

Tycho SNR was observed by XMM-Newton (Jansen et al. 2001) on 2000 June 29 with the two X-ray instruments EPIC (European Photon Imaging Camera; Strüder et al. 2001; Turner et al. 2001) and RGS (Reflection Grating Spectrometer; den Herder et al. 2001). After selecting good time intervals (when the light curve in background regions is stable, i.e. uncontaminated by flaring events), the available exposure time was $12 \mathrm{ks}$ for each EPIC MOS camera and $15 \mathrm{ks}$ for the EPIC PN camera. Due to the high signal-to-noise $(S / N)$ ratio of the RGS spectra of Tycho and the smaller sensitivity of the RGS instruments to background variations, no time-selection was applied to the RGS data, resulting in a total of $45 \mathrm{ks}$ of exposure time. The X-ray image of Tycho is entirely contained within the central CCD of each of the MOS cameras whereas in the PN camera it is spread over 6 CCDs, resulting in data gaps between each CCD. For simplicity, therefore, we have only used the EPIC MOS data in our spatial analysis. 

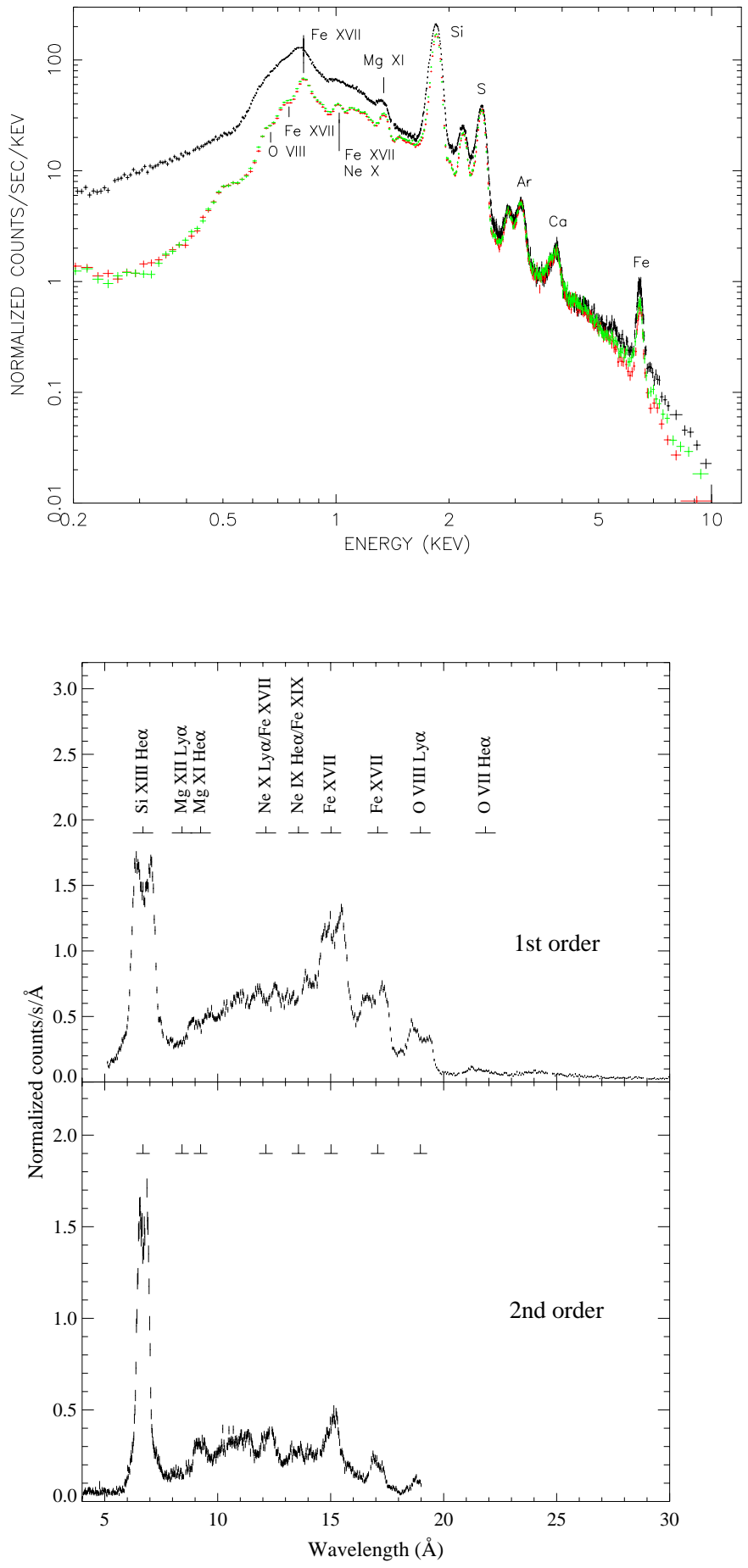

Fig. 1. Top: EPIC spectra integrated over the whole remnant obtained with the MOS 1 (green), MOS 2 (red) and PN (black) camera. Bottom: RGS 1 spectrum of a limited region of Tycho (see Fig. 2) with order 1 and order 2. The spatial extent of the SNR dominates the line profiles

The three EPIC spectra (MOS 1, MOS 2 and PN), integrated over the entire remnant, are shown in Fig. 1, with the RGS spectrum obtained on a region smaller than Tycho (the FOV is shown in Fig. 2).
The most prominent lines in the EPIC spectra are the He $\alpha$ lines of magnesium, silicon, sulphur, argon, calcium, iron and an Fe XVII line in the Fe L complex. With the RGS, the remnant has been detected up to the 5th order, however with a low $S / N$ ratio from the third order. Emission lines from Si XIII He $\alpha, \operatorname{Mg}$ XI He $\alpha, \operatorname{Nex} \operatorname{Ly} \alpha$, NeIX He $\alpha$, two FexviI lines (at $15 \AA$ and $17 \AA$ ) and $\mathrm{O}$ VIII Ly $\alpha$ have been identified. The structure seen both in EPIC and RGS spectra between the magnesium and neon lines $(10 \AA$ and $12 \AA)$ is attributed to Fe L shell lines. Both RGS data sets were analyzed and are fully consistent with each other.

\section{Elemental structure in the ejecta}

The bright emission lines of heavy elements (silicon, sulphur, iron) in the spectrum of the entire remnant originate from shocked material within the supernova ejecta.

We have created images selecting photons in narrow energy bands corresponding to the main line features. For the maps of Si XIII and Fe XVII, we did not substract the continuum: these two lines have the highest $S / N$ ratio above the continuum. For the lower $S / N$ ratio Fe $\mathrm{K} \alpha$ line, where the spectral shape is steep, the continuum does need to be subtracted. This was done by using the overall global spectrum to derive the ratio of the continuum at the line energy to the continuum in an adjacent energy band, free of contamination by line emission. In each spatial bin, the continuum at the line was estimated using the flux in the adjacent energy band and the previously defined ratio.

To complement this image approach, we present azimuthally averaged radial profiles of the deconvolved line images. For the MOS1 and MOS2 cameras, the FWHM of the point spread function is $4.4^{\prime \prime}$ at $1500 \mathrm{eV}$ and is slightly larger at higher energy (Aschenbach et al. 2000; Gondoin et al. 2000).

We have used a standard Van Cittert deconvolution algorithm (Starck et al. 1998) to reconstruct the images within typically 20 iterations.

\subsection{Large scale structure}

The $15 \AA$ Fe XVII line image is shown in Fig. 2, overlaid with the Si XIII He $\alpha$ contours. Overall, the spatial distribution is very similar, although Fig. 2 shows that the image, as well as the radial profile, in the SixIII line is probably slightly peaked exterior to the Fe XVII line image. The good correlation between the emission from iron and silicon implies that the outer layers of the supernova have been at least partially mixed. This is predicted by numerical hydrodynamical modeling of the global X-ray spectrum of Tycho (Itoh et al. 1988; Brinkmann et al. 1989).

In Fig. 3, we show again the $15 \AA$ Fe XVII line image, overlaid with the contours in the continuum subtracted $\mathrm{Fe} \mathrm{K} \alpha$ line image.

There is an overall agreement between the line images, with spherical emission brightest in the northwest, 

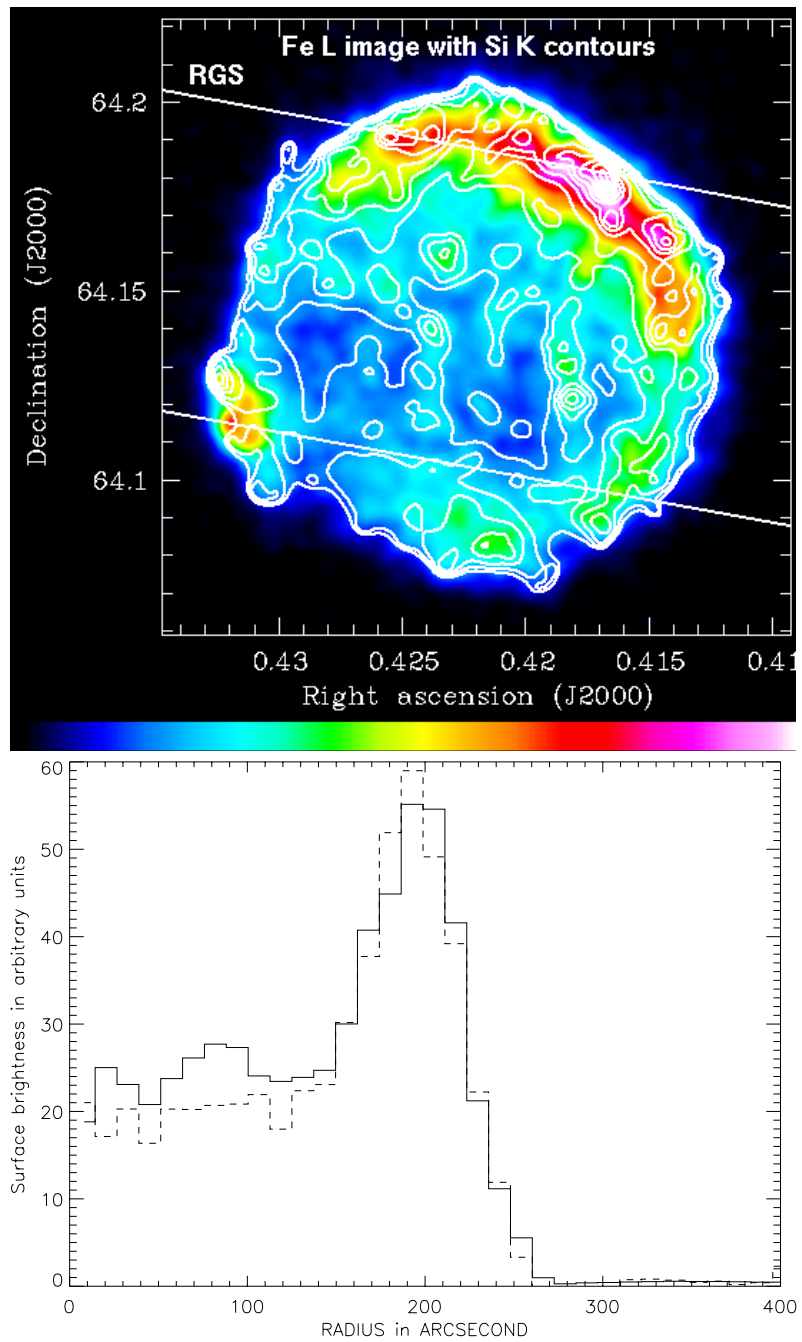

Fig. 2. Top: contours of the Si XIII triplet blend (1670-2000 eV) superimposed on the EPIC MOS image in the FexVII $(\lambda=$ $15 \AA, 775-855 \mathrm{eV}$ ) line. The RGS field of view is overlaid and the dispersion axis is parallel to the solid white lines. The Right Ascension is in decimal degrees. Bottom: comparison of the azimuthally averaged radial profiles of the deconvolved images in the Si K (solid line) and Fe XVII lines (dashed line)

faintest in the east and intermediate in the south sectors as for Si XIII. Both Fe lines exhibit strong emission in the southern part of the southeast knots, and a faint contribution to the northern part for Fe XVII. The comparison of the radial profiles and of the images demonstrates that the spatial distribution of the Fe $\mathrm{K} \alpha$ emission is broader, and peaks at smaller radius, than the Fe XVII emission, as was first suggested by Hwang \& Gotthelf (1997), based on $A S C A$ deconvolved narrow band images.

The fact that the Si XIII He $\alpha$ and Fe XVII images correlate well overall, whilst the $\mathrm{Fe} \mathrm{L}$ and $\mathrm{Fe} \mathrm{K}$ lines of iron are clearly displaced, favors the interpretation of a spatial structure in the temperature and ionization timescale in the shocked ejecta. This is indeed what is expected from hydrodynamical evolution models of young supernova remnants (see for example Chevalier 1982; Decourchelle \& Ballet 1994). Although a spatial segregation of the
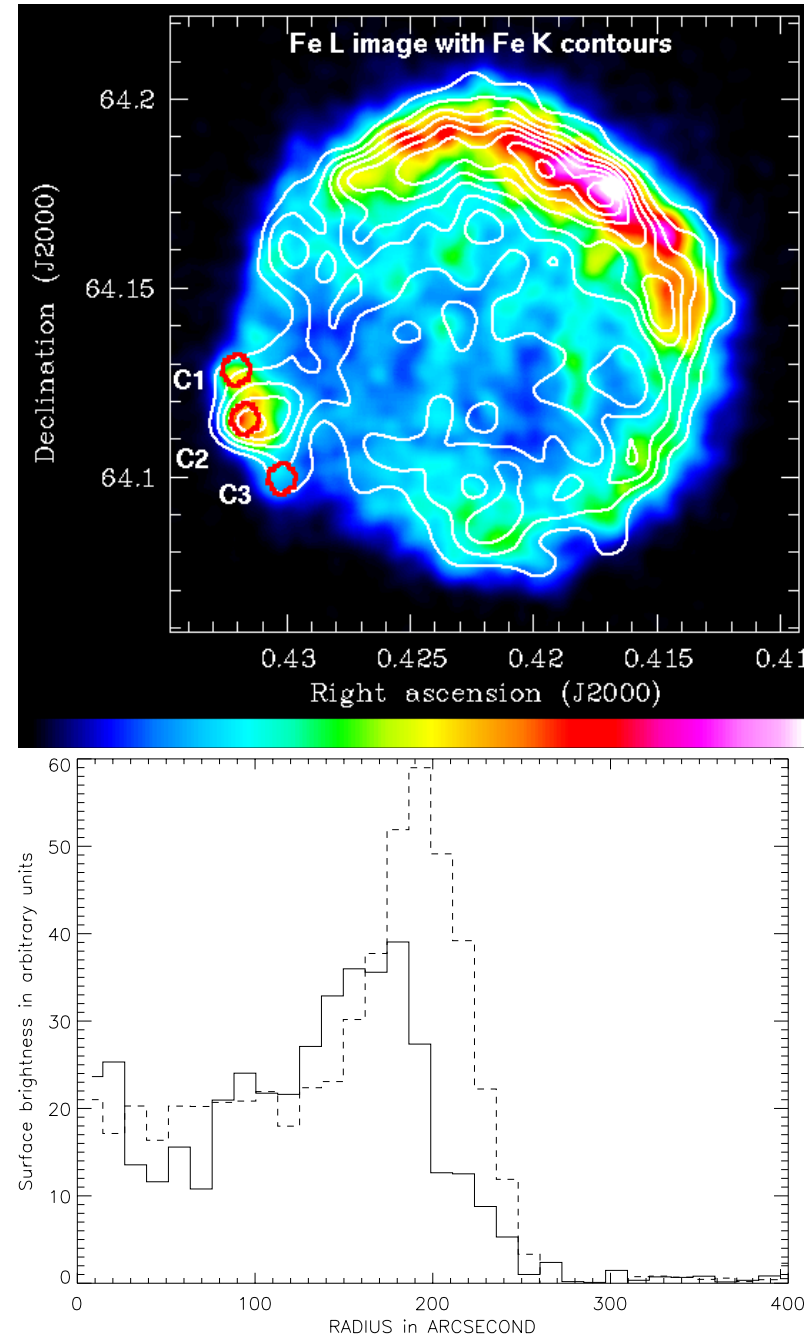

Fig. 3. Top: contours of the Fe $\mathrm{K}$ line $(6200-6600 \mathrm{eV})$ image superimposed on the EPIC MOS image in the FeXVII line. Fields C1, C2 and C3 are shown with red solid lines. Bottom: comparison of the radial profiles of the deconvolved images in the Fe K (solid line) and Fe XvII lines (dashed line)

elements is possible, depending on the degree of mixing, a structure in temperature and/or ionization stage is in most cases unavoidable and depends on the initial density profiles of the supernova.

The Fe K line corresponds to higher temperature material (at least twice higher) and lower ionization timescale than the Fe L and silicon emission (Hwang et al. 1998). This indicates that the temperature in the inner shocked ejecta is higher with a lower ionization age than the outer shocked ejecta region. Such profiles of temperature and ionization age are those qualitatively expected from the evolution of a supernova remnant of $1.4 M_{\odot}$ in a uniform interstellar medium, when the reverse shock is propagating, from an outer power-law density profile, into the inner plateau region of the ejecta. For an explosion energy of $10^{51}$ ergs and an ambient density of $0.35 \mathrm{amu} \mathrm{cm}^{-3}$, the reverse shock, at the age of Tycho, has already entered the central plateau (Decourchelle 1994). The dense shocked ejecta region (colder and older), close to the 
contact discontinuity, is the result of the interaction of a power-law density ejecta profile with the ISM (Chevalier 1982) at the beginning of the evolution. When the reverse shock reaches the central plateau, the density begins to decrease in the ejecta, the temperature increases and the X-ray emissivity diminishes (see for example Fig. 6 in Band \& Liang 1988). This is qualitatively what we observe in Tycho with the inner Fe $\mathrm{K}$ line in a region with fainter emission.

Standard models of the type Ia supernova explosion, such as the W7 model (Nomoto et al. 1984), give outer density profiles in the ejecta closer to an exponential decline than to a power law. However, the characteristic temperature profiles for exponential ejecta expanding into a constant density ambient medium are flatter than the temperature gradient required in Tycho, unless a circumstellar medium is invoked (Dwarkadas \& Chevalier 1998). Note however that for stratified ejecta, the heavier element layers would have a higher temperature because of the lower number of electrons for the same mass density.

\subsection{Small scale inhomogeneities}

A more careful inspection of the comparison between the silicon and iron images (see Fig. 2) shows that of the southeastern knots (Vancura et al. 1995) the northern one is brightest in the Si XIII line, whereas the southern one is brightest in the Fe XVII line.

Figure 4 (top panel) shows the MOS 1 and MOS 2 spectra of the three southeastern knots, marked C1, C2 and C3 in Fig. 3. We have extracted spectra from these identically sized regions and fit them with a two component non-equilibrium model (with variable abundances for the first component). A Gaussian line at $3.1 \mathrm{keV}$ was added to the model to account for the argon emission line, which is missing in the non-equilibrium model VNEI within XSPEC (Arnaud 1996).

The first component is at a relatively low temperature $\left(T_{1}\right)$ and accounts for the line emission which is the focus of this discussion, whilst the second component assumes solar abundances, is at a high temperature and accounts for the high energy continuum. We assume no hydrogen and helium in the shocked SN Ia ejecta, and solar values for carbon and nitrogen.

The three different spectra can be simultaneously fitted with the same temperature $\left(k T_{1}=0.62 \pm 0.02 \mathrm{keV}\right)$, ionization timescale $\left(1.210^{11} \mathrm{~s} \mathrm{~cm}^{-3}\right)$ and hydrogen column density $\left(0.8310^{22} \mathrm{~cm}^{-2}\right)$ and a relatively good fit is achieved ( $\chi^{2}=1183 / 721$ dof $)$. However, there are, between the knots, clear variations of the abundances of silicon, sulphur and iron, which are given in Table 1.

The spectral analysis demonstrates that the different emission structures seen in the southeastern knots are primarily due to spatial variations of the relative abundances of silicon, sulphur and iron, rather than temperature and ionization timescale effects. The relative abundance of sulphur to silicon is roughly constant in these three knots,

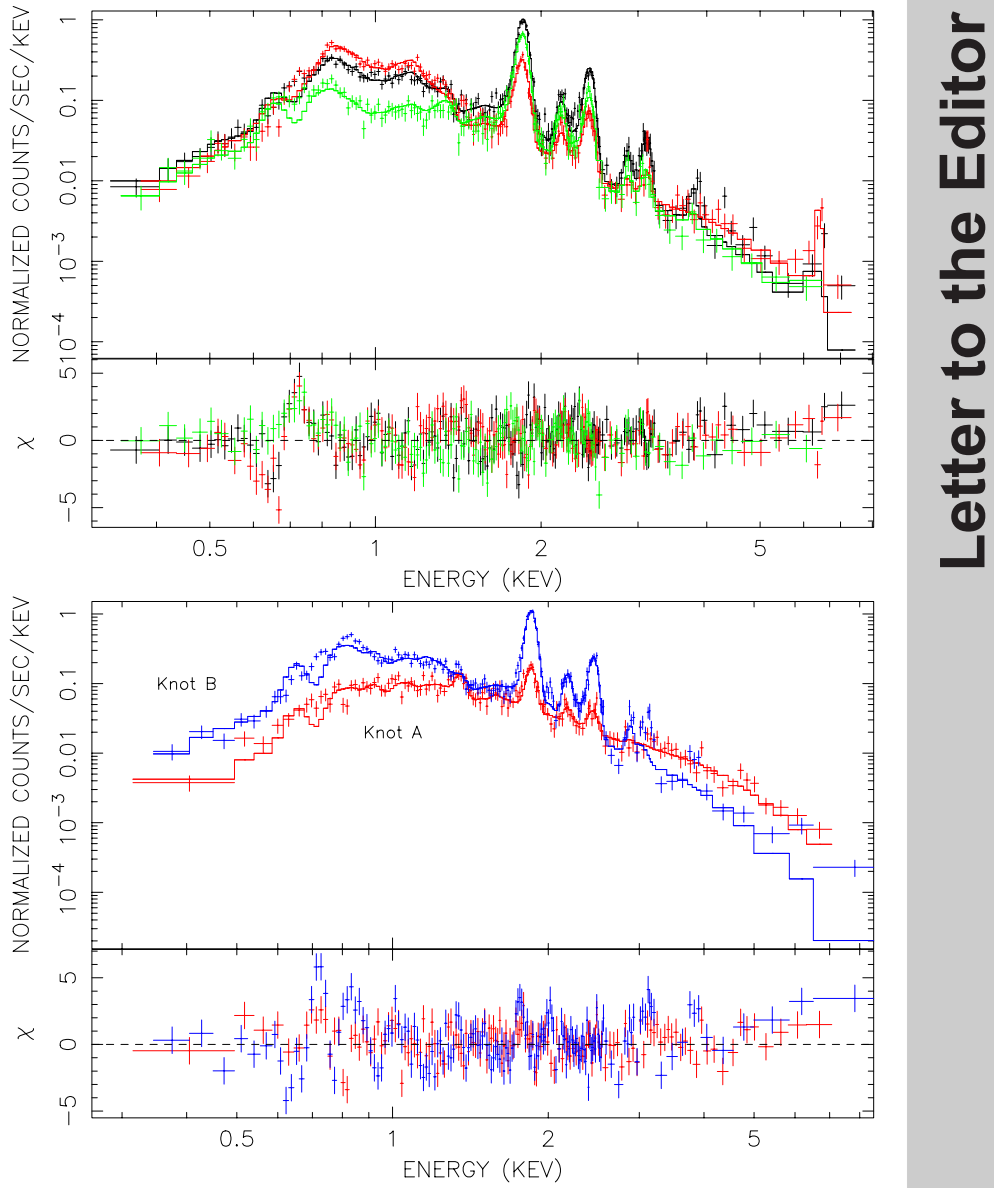

Fig. 4. Top: MOS 1 and MOS 2 Spectra of the three southeastern knots C1 (red), C2 (black) and C3 (green). Bottom: spectra of a strong continuum region (Knot $\mathrm{A}$ in red) and a line dominated region (Knot $\mathrm{B}$ in blue). The regions $\mathrm{A}$ and $\mathrm{B}$ are shown in Fig. 5

Table 1. Derived abundances (relative to the solar photospheric values) in the knots

\begin{tabular}{llllll}
\hline & C1 & C2 & C3 & A & B \\
\hline Si & 2.1 & 1.1 & 1.6 & 1.0 & 4.2 \\
S & 4.6 & 2.2 & 2.1 & 1.1 & 6.9 \\
$\mathrm{Fe}$ & 0.2 & 0.4 & 0.06 & 0.1 & 0.7 \\
\hline
\end{tabular}

while that of iron is varying. Knot C1 has the largest silicon and sulphur abundance, whereas knot C3 has almost no iron. In this particular knot C3, almost a factor of 10 less iron has protruded into the silicon layer compared with the adjacent knot $\mathrm{C} 2$. However, the southeastern knots are the only place where such a relative variation of silicon to iron is unambigously observed. As shown in Fig. 2 and discussed in Sect. 3, there is otherwise a good agreement between the images in Fe XVII and in Si XIII for the whole remnant. These knots resemble to some extent the silicon rich shrapnels seen in Vela (Aschenbach et al. 1995; Tsunemi et al. 1999). 


\section{High-energy continuum and radio emission}

The high-energy continuum image in the 4500-5800 eV band is shown in Fig. 5, overlaid with the Si XIII emission contours. The image in the continuum is more regular and extended than in the silicon line, which shows irregular outer contours, which can be attributed to RayleighTaylor instabilities at the contact discontinuity between the ejecta material and the ambient medium (see Fig. 6). The extent of the continuum emission corresponds well with the radio VLA image at $22 \mathrm{~cm}$ (Dickel et al. 1991) and gives the position of the outer blast wave. This is shown in Fig. 5, where the radio image is overlaid with the contours of the continuum emission. The regular spherical emission of the continuum, unlike the emission line images, may indicate that the ambient medium surrounding Tycho was fairly homogeneous, although it has been recently interacting on its eastern edge with $\mathrm{H}$ I clouds (Reynoso et al. 1999). The more asymmetric shape in the line emission may be related to azimuthal variations of the heavy element distribution in the ejecta.

The radial profiles of the $\mathrm{X}$-ray continuum and radio emission are shown in the bottom panel of Fig. 5. The peak of the continuum emission is the outermost and is interpreted as emission from the shocked interstellar medium. The radio emission extends as far as the continuum but the profile peaks at about the same radius as the Si emission (Fig. 2). For comparison the profile of the Fe K emission is also shown and clearly peaks at a much smaller radius than the other components.

In addition to a regular smooth profile, the continuum map shows striking bright knots on the eastern edge, as well as on the diametrically opposite side of the remnant. These features are lying inner to the continuum edge, they have no clear counterpart in the line images, nor in the radio. However, the brightest continuum knot (eastern edge) lies very close to the two brightest radio peaks, which exhibit the flattest spectral index of the remnant (Filament I, in Katz-Stone et al. 2000), while the southwestern continuum knots lie along a radio structure, which also has a flat spectral index (Filament A, in Katz-Stone et al. 2000). The eastern knot has the lowest radio expansion in Tycho (Reynoso et al. 1997, see Hughes 2000 for the X-ray expansion rate), and is close to one of the brightest optical filaments (Kamper \& Van den Bergh 1978; Smith et al. 1991), associated with infrared emission at 10.7-12 micron (Douvion et al. 2000). A recent H I $21 \mathrm{~cm}$ study of the environs of Tycho has found a strong H I absorption feature, corresponding to a cloud of density $160-325 \mathrm{~cm}^{-3}$ on the eastern edge (Reynoso et al. 1999).

The bright continuum knot lies at the periphery of the line emission image, whilst being well inside the continuum emission. The comparison of the deconvolved silicon image with the radio image shows that the eastern side is the only place where the radio and silicon boundaries overlap and where such a strong X-ray continuum is seen (see cross mark in Fig. 6). The two brightest radio knots coincide with similar features in the silicon image. This indicates
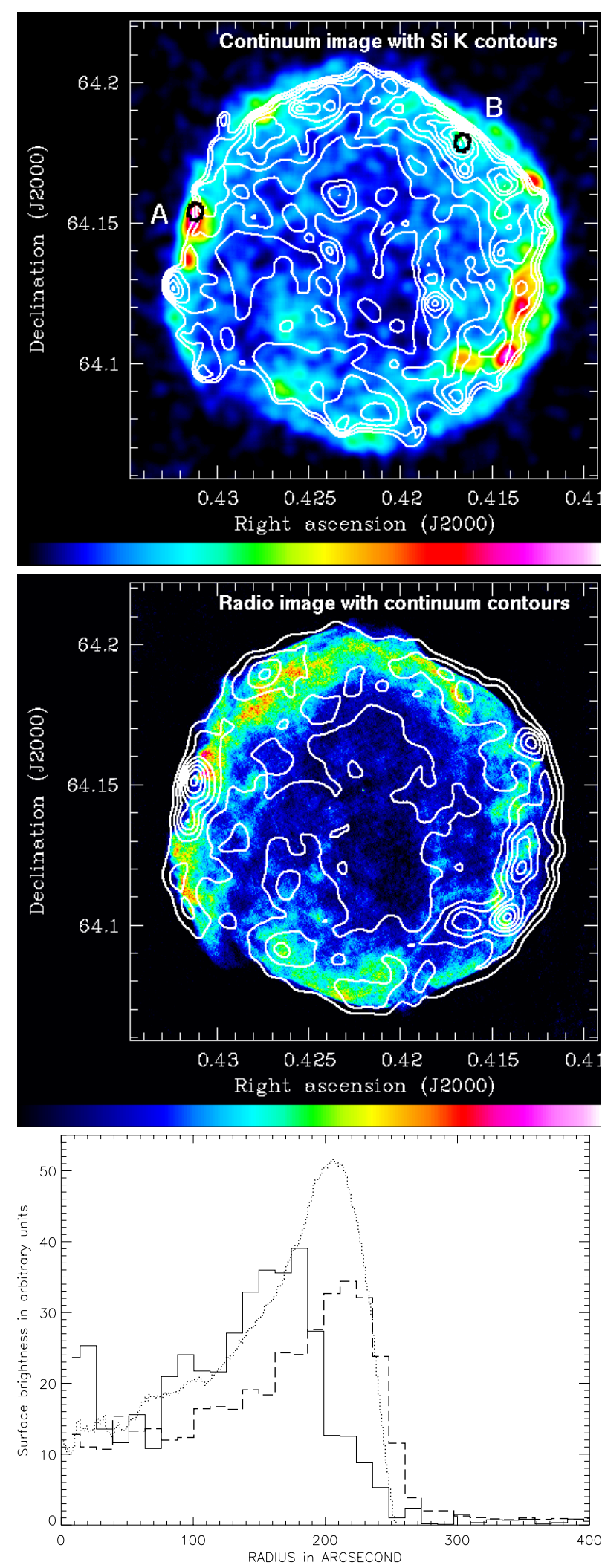

Fig. 5. Top: high energy continuum image (4500-5800 eV) overlaid with Si XIII contours. Fields A and B are shown with black solid lines. Middle: VLA $22 \mathrm{~cm}$ radio map overlaid with high energy continuum contours. Bottom: comparison of the three corresponding radial profiles: radio (dotted), high energy continuum (dashed) and Fe K line (solid line). 


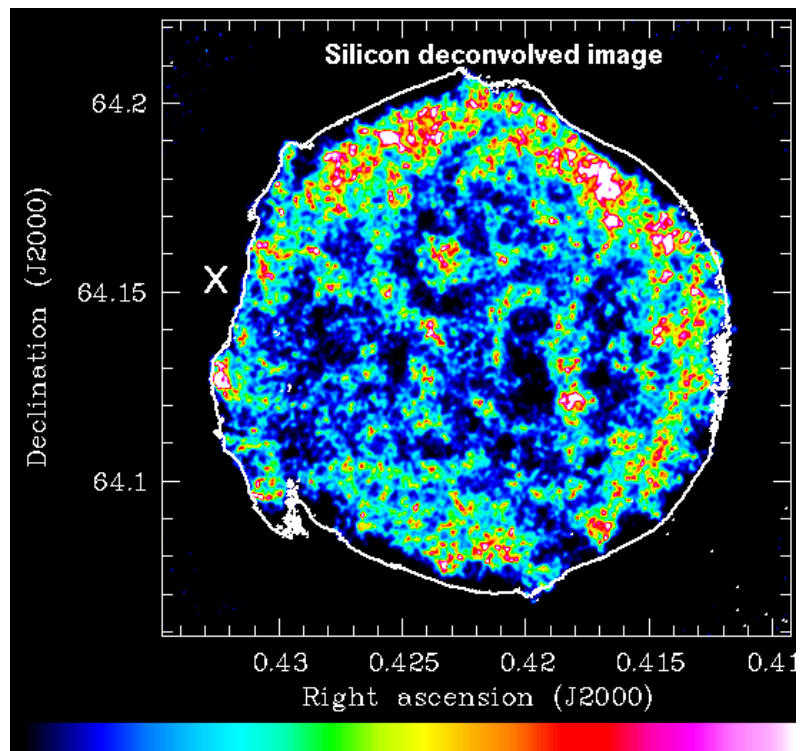

Fig. 6. Deconvolved silicon image overlaid with outer contour of the radio image from Dickel et al. (1991)

that they are related to ejecta material, with structures characteristic of Rayleigh-Taylor instabilities as argued by Velasquez et al. (1998), based on VLA radio images with a resolution of $1^{\prime \prime}$.

Jun \& Norman (1996) have shown that RayleighTaylor instabilities amplify strongly the ambient local magnetic field around the dense features of the instability by stretching, winding and compressing the magnetic field lines. Assuming an external magnetic field tangential to the shock front, their simulations account for the observed radial vector polarization observed in the remnant (Dickel et al. 1991; Reynoso et al. 1997) and predict an amplification of the magnetic field in front of such RayleighTaylor features, which would explain the fact that these structures are seen both in radio (amplified synchrotron emission around these instabilities) and in X-rays (thermal emission of the dense knots).

The strong continuum knot might be the result of the interaction of the eastern edge with $\mathrm{H}$ I clouds, leading either to higher densities and magnetic field strengths (hence stronger nonthermal emission), or to a reflected shock in front of the cloud, which heats and compresses a second time the ISM (hence leading to an increase in the thermal bremsstrahlung emission at high energy). However, no $\mathrm{HI}$ features have been observed in the diametrically opposite edge, where a strong X-ray continuum is also observed.

The bipolar symmetry of the regions emitting this strong X-ray continuum may be an indication of particle acceleration with an external magnetic field tangential to the shock front in these regions as in the case of SN 1006 (Reynolds 1996, 1998).

Figure 4 (bottom panel) shows the MOS 1 and MOS 2 spectra of Knot A and Knot B (highlighted in Fig. 5). The spectrum of Knot A features a strong high energy continuum, whereas the spectrum of Knot B is typical of the brightest silicon dominated regions. We have used a single non-equilibrium model to fit the data (VNEI, XSPEC). The silicon and sulphur abundances in Knot A are close to solar, but all other elements have much lower values, in particular iron (see Table 1). The temperature is more than a factor 2 higher in Knot $\mathrm{A}(k T=2.3 \pm 0.3 \mathrm{keV})$ for an ionization timescale $\left(n_{\mathrm{e}} t \simeq 1.4 \pm 0.410^{10} \mathrm{~s} \mathrm{~cm}^{-3}\right)$ three times shorter than in Knot B $(k T=0.9 \pm 0.1 \mathrm{keV}$, $\left.n_{\mathrm{e}} t \simeq 4.7 \pm 0.310^{10} \mathrm{~s} \mathrm{~cm}^{-3}\right)$.

Both the narrow band images and the spectral analysis indicate that the continuum dominated emission most probably arises from the shocked ambient medium. We cannot, however, unambiguously identify the nature of this emission. The signature of a thermal plasma in this spectrum comes primarily from the silicon and sulphur lines, although they can also correspond to spatial contamination (due to the PSF) from the much brighter surrounding ejecta emission. A deeper systematic analysis of all the strong continuum knots is required to determine precisely their origin, which is beyond the scope of this paper.

\section{Conclusion}

We present the first results obtained from the XMMNewton observation of the Tycho supernova remnant.

- The good correlation between the images in the Si XIII K line and FexVII L line implies that some fraction of the inner iron layer has been well mixed with the outer silicon layer;

- The fact that the Fe K line emission peaks distinctly at a smaller radius than the Fe $\mathrm{L}$ line emission is a clear indication that there is a spatial structure in the temperature in the ejecta, with higher temperature towards the reverse shock. This is coherent with the propagation of the reverse shock in the inner plateau of the ejecta;

- The silicon image correlates well at small scales with the radio image, and probably marks the contact discontinuity, distorted by Rayleigh-Taylor instabilities;

- The high-energy continuum, which is regular overall, peaks just behind the shock front, and is attributed to the emission from the shocked ambient medium;

- In addition to this regular continuum emission, we have discovered particularly bright and hard continuum knots on the eastern and western edges.

Acknowledgements. We would like to warmly thank John Dickel for providing us with the $22 \mathrm{~cm}$ VLA radio image of Tycho as well as Philippe Gondoin for providing us with the PSF at different energies. M. A. acknowledges support from the Swiss National Science Foundation (grants 2100-049343 and 2000-058827), from the Swiss Academy of Sciences and from the Swiss Commission for Space Research.

\section{References}

Arnaud, K. A. 1996, ASP Conf. Ser. 101, Astronomical Data Analysis Software and Systems V, ed. G. Jacoby, \& J. Barnes (San Francisco: ASP), 17 
Aschenbach, B., Briel, U., Haberl, F., et al. 2000, Proc. SPIE [astro-ph/0007256]

Aschenbach, B., Egger, R., \& Trümper, J. 1995, Nat, 373, 587

Baade, W. 1945, ApJ, 102, 309

Band, D. L., \& Liang, E. P. 1988, ApJ, 334, 266

Brinkmann, W., Fink, H. H., Smith, A., \& Haberl, F. 1989, A\&A, 221, 385

Chevalier, R. A. 1982, ApJ, 258, 790

Decourchelle, A. 1994, Ph.D. Thesis, University of Paris VII

Decourchelle, A., \& Ballet, J. 1994, A\&A, 287, 206

den Herder, J. W., Brinkman, A. C., Kahn, S. M., et al. 2001, A\&A, 365, L7

Douvion, T., Lagage P. O., Cesarsky, C. J., \& Dwek, E. 2000, A\&A, in preparation

Dickel, J. R., van Breugel, W. J. M., \& Strom, R. G. 1991, AJ, 101,2151

Dwarkadas, V. V., \& Chevalier, R. A. 1998, ApJ, 497, 807

Gondoin, P., Aschenbach, B., Erd, C., et al. 2000, Proc. SPIE

Hughes, J. P. 2000, ApJL, in press [astro-ph/0010122]

Hwang, U., \& Gotthelf, E. V. 1997, ApJ, 475, 665

Hwang, U., Hughes, J. P., \& Petre, R. 1998, ApJ, 497, 833

Itoh, H., Masai, K., \& Nomoto, K. 1988, ApJ, 334, 279

Jansen, F., Lumb, D., Altieri, B., et al. 2001, A\&A, 365, L1

Jun, B.-I., \& Norman, M. L. 1996, ApJ, 465, 800
Kamper, K. W., \& van den Bergh, S. 1978, ApJ, 224, 85

Katz-Stone, D. M., Kassim, N. E., Lazio, T. J. W., \& O'Donnell, R. 2000, ApJ, 529, 453

Nomoto, K., Thielemann, F.-K., \& Yokoi, K. 1984, ApJ, 286, 644

Reynolds, S. P. 1996, ApJL, 459, 13

Reynolds, S. P. 1998, ApJ, 493, 375

Reynoso, E. M., Moffett, D. A., Goss, et al. 1997, ApJ, 491, 816

Reynoso, E. M., Velásquez, P. F., Dubner, G. M., \& Goss, W. M. 1999, AJ, 117, 1827

Smith, R. C., Kirshner, Robert P., et al. 1991, ApJ, 375, 652

Starck, J. L., Murtagh, F., \& Bijaoui, A. 1998 (Cambridge University Press, Cambridge)

Strüder, L., Briel, U. G., Dennerl, K., et al. 2001, A\&A, 365, L18

Tsunemi, H., Miyata, E., \& Aschenbach, B. 1999, PASJ, 51, 711

Turner, M. J. L., Abbey, A., Arnaud, M., et al. 2001, A\&A, $365, \mathrm{~L} 27$

Vancura, O., Gorenstein, P., \& Hughes, J. P. 1995, ApJ, 441, 680

Velásquez, P. F., Gómez, D. O., Dubner, G. M., et al. 1998, A\&A, 334, 1060 